\title{
Recent Advances in Childhood Arterial Ischemic Stroke
}

\author{
Christine K. Fox • Heather J. Fullerton
}

Published online: 15 May 2010

(C) The Author(s) 2010. This article is published with open access at Springerlink.com

\begin{abstract}
Although many underlying diseases have been reported in the setting of childhood arterial ischemic stroke, emerging research demonstrates that non-atherosclerotic intracerebral arteriopathies in otherwise healthy children are prevalent. Minor infections may play a role in arteriopathies that have no other apparent underlying cause. Although stroke in childhood differs in many aspects from adult stroke, few systematic studies specific to pediatrics are available to inform stroke management. Treatment trials of pediatric stroke are required to determine the best strategies for acute treatment and secondary stroke prevention. The high cost of pediatric stroke to children, families, and society demands further study of its risk factors, management, and outcomes. This review focuses on the recent findings in childhood arterial ischemic stroke.
\end{abstract}

Keywords Childhood stroke - Arterial ischemic stroke . Cerebral arteriopathy $\cdot$ Thrombolysis $\cdot$ Stroke outcomes

\section{Introduction}

Tremendous efforts have been made to increase public recognition of adult stroke, yet pediatric stroke remains under-recognized even among health care providers. However, pediatric stroke is an important cause of lifelong disability. A recent population-based study of stroke in

C. K. Fox $(\bowtie) \cdot H$. J. Fullerton

University of California, San Francisco,

Box 0114, 505 Parnassus Avenue, Moffitt S798,

San Francisco, CA 94143-0114, USA

e-mail: foxc@neuropeds.ucsf.edu

H. J. Fullerton

e-mail: fullertonh@neuropeds.ucsf.edu children less than 20 years of age estimates an annual rate of 2.4 arterial ischemic strokes per 100,000 children [1]. Large-scale studies of pediatric stroke have begun to advance our understanding of underlying risk factors, but little research is currently available to guide decision making about acute management and secondary stroke prevention. Many of these children are left with permanent neurologic deficits and epilepsy, and pediatric stroke incurs a high cost to families and societies. Although pediatric stroke includes arterial ischemic stroke, hemorrhagic stroke, and venous thrombosis, hemorrhage and venous thromboses are beyond the scope of this brief update.

\section{Risk Factors for Arterial Ischemic Stroke: The Importance of Arteriopathies}

Overall, presumptive risk factors for arterial ischemic stroke differ in children compared with adults. Whereas adult risk factors are primarily related to arrhythmias and obstructive atherosclerotic arteriopathies, these are rarely thought to be related to stroke in children. Also in contrast to adults, socioeconomic status has not been shown to be associated with an increased stroke risk in children [2]. Interestingly, just as gender differences are found in adult stroke studies, several pediatric studies have shown a male predominance in children with a stroke $[2,3]$. Among boys, elevated testosterone levels were independently associated with stroke risk [4]. The mechanism of this association is still unknown. In the International Pediatric Stroke Study (IPSS) and other studies of pediatric stroke, a wide range of underlying systemic factors were reported in the setting of childhood stroke, particularly sickle cell disease, cardiac disorders, trauma, and major infections such as meningitis, sepsis, and encephalitis [2]. However, in the majority of the 
children, no underlying systemic disease is found. With advances in neuroimaging, cerebral arteriopathies are increasingly recognized as one of the major causes of childhood stroke in otherwise healthy children $[6,7 \bullet]$. Although the importance of arteriopathies is highlighted in this review, advances in understanding of sickle cell disease, cardiac disease, and prothrombotic disorders are also discussed.

Arteriopathies are an Important Underlying Cause of Childhood Arterial Ischemic Stroke

The IPSS recently reported on presumptive risk factors in their case series of 667 children from five continents that have had a stroke [5]. In the study, $53 \%$ of the children who had vascular imaging were found to have an arteriopathy [7•]. Some arteriopathies are due to well-recognized causes such as arterial dissection, moyamoya syndrome or disease, connective tissue disorders, and sickle cell disease. Late effects of radiation to the head and neck in childhood cancer survivors have also been associated with stroke due to cerebrovascular disease, and children with neurofibromatosis can have complications of a cerebral arteriopathy [8]. However, in up to $30 \%$ of children presenting with a first arterial ischemic stroke, a focal arterial stenosis is identified but none of the classic underlying causes are discovered.

The term "transient cerebral arteriopathy" (TCA) was first used to describe a common course of cerebrovascular pathology in a group of children with ischemic stroke who received serial imaging studies [9]. In this group, focal stenoses or segmental narrowing of the cerebral arterial wall, characteristically involving the distal internal cerebral artery, proximal middle cerebral artery, and sometimes the proximal anterior cerebral artery, were followed over time with serial angiograms (Fig. 1). The term "transient" was used because of the monophasic progression of cerebrovascular disease. In children with TCA, the arterial stenosis and narrowing is strictly unilateral and may initially worsen. In some cases, no abnormality is noted on initial vascular imaging, but stenosis or occlusion is seen on follow-up imaging within the first few months. However, after the first 6 months, the arteriopathy stabilizes and sometimes improves. In some sense, the term "transient" cerebral arteriopathy is somewhat misleading. Although monophasic in the progression of stenosis, in many cases the arterial narrowing persists on long-term follow-up imaging.

On initial imaging studies, TCA cannot be distinguished from a progressive arteriopathy such as moyamoya disease or a vasculitis that presents unilaterally. Worsening after 6 months or bilateral involvement suggests an arteriopathy other than TCA, with a different course and prognosis. In

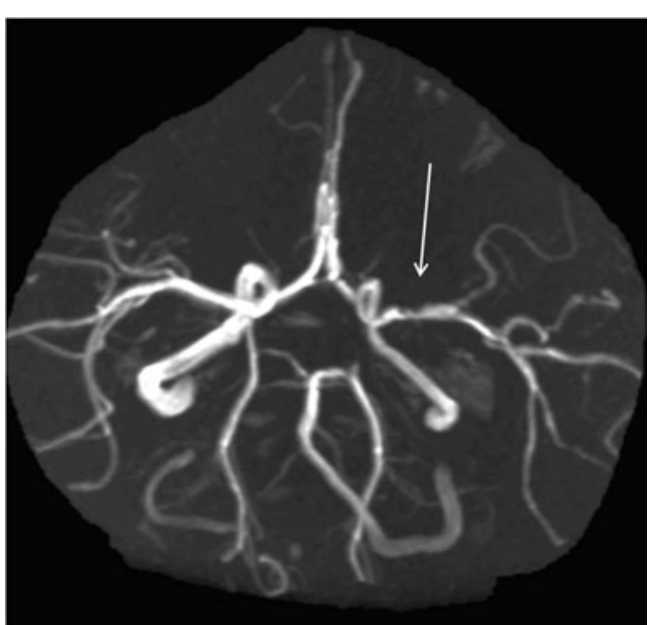

Fig. 1 A magnetic resonance angiography (MRA) image taken 7 months after a stroke in a 7-year-old girl demonstrates a persistent, stable, unilateral stenosis of the left proximal middle cerebral artery. This imaging is typical of "transient cerebral arteriopathy," a monophasic focal cerebral arteriopathy observed in many previously healthy children with stroke. The stenosis shown in this image (arrow) was essentially unchanged from the MRA done acutely after the stroke

recognition of the limitations of classifying arteriopathies on initial imaging, the IPSS has recently suggested the term "focal cerebral arteriopathy" of childhood (FCA) to describe children with unilateral arterial stenosis without an apparent underlying cause at presentation [7•]. Both the terms TCA and FCA are simply descriptive and do not imply a specific pathophysiology; however, these arteriopathies are clinically important because they indicate an increased risk for recurrent stroke $[6,10,11]$. In all children who have had a stroke, vascular imaging of intracranial and neck vessels is imperative to detect arteriopathies, with magnetic resonance angiography (MRA) as the preferred modality. However, if interventional neuroradiologists who have an expertise in pediatrics are available, conventional angiography can be considered in cases in which diagnosis remains unclear on MRA. In addition, even in children with a stable clinical course, MRI and vascular imaging should be repeated 3-6 months after the acute stroke and again 1 year later to detect development of or changes in arteriopathies, as well as to look for new subclinical infarcts.

\section{Recent Infection is a Risk Factor for Focal Cerebral Arteriopathy in Childhood}

Currently, the underlying mechanisms for these unilateral, monophasic arteriopathies are poorly understood, and studies are ongoing to elucidate their pathophysiology. The role of infection as a part of the underlying vascular pathology is of particular current interest. The link between childhood stroke and infection was first noted because of its 
temporal association with varicella infection; post-varicella angiopathy has been well described as a cause for ischemic stroke in both adults and children [12, 13]. Importantly, no association has been found with varicella vaccination [14]. Multiple viruses other than varicella have been linked to case reports of arteriopathies and stroke in children. Strengthening the infectious hypothesis, the IPSS reported finding an association between childhood arteriopathies and recent minor infections in their large cohort of children with ischemic stroke [7•]. In the study, $25 \%$ of the children were classified as having FCA, higher in frequency than either moyamoya (22\%) or dissection $(20 \%)$. Predictors of overall arteriopathies were early school age (5-9 years), recent upper respiratory infections, and sickle cell disease. The only predictor of FCA was a recent upper respiratory infection (odds ratio $[\mathrm{OR}]=2.1 ; 95 \% \mathrm{CI}, 1.01-4.38$ ). The role of minor infections in the pathogenesis of stroke has been suggested either as a cause of direct vascular injury, or by inducing a systemic prothrombotic state, or through both mechanisms. However, minor infections are extremely common in young children, and yet most children do not have strokes. More study is needed to clarify how infection contributes to arteriopathies and stroke.

Transcranial Doppler Screening Effectively Reduces Stroke Risk in Children with Sickle Cell Disease, but Barriers to Care Persist

Children with sickle cell disease comprise another important group with a high risk of arteriopathies and stroke. Prior to modern primary prevention strategies, up to $11 \%$ of children with sickle cell anemia had a clinical stroke by the age of 20 years [15]. In 1992, transcranial Doppler (TCD) was found to be effective in identifying patients with sickle cell disease at high risk for stroke [16]. The Stroke Prevention Trial in Sickle Cell Anemia (STOP) established that chronic transfusion decreased the risk of stroke by $90 \%$ in children with abnormal TCD results [17], leading to recommendations for chronic transfusion for prevention of stroke in children deemed at high risk. However, TCD screening in sickle cell disease continues to be examined for feasibility and efficacy internationally [18, 19], and barriers to care persist. A recent study of children in northern California showed the rate of stroke in children with sickle cell disease has dropped by more than half the rate prior to 1998 , but also demonstrated that children living farther from a vascular laboratory are less likely to be screened [20]. Finally, although stroke risk in people with sickle cell disease is usually attributed to red cell sickling and vasculopathy of large intracranial vessels, new concerns have recently been raised for an increased risk for cardioembolic stroke in these children because of the potential for increased right heart pressure and right-to-left shunting in the setting of a prothrombotic state [21].This suggests the intracardiac shunts in children with sickle cell disease should be further studied as a potentially modifiable cause of stroke. Current American Heart Association stroke prevention screening recommendations in sickle cell disease are for annual TCD monitoring of children with normal TCD velocities $(\leq 170 \mathrm{~cm} / \mathrm{s})$, with consideration of more frequent monitoring in children 2 to 10 years of age. Children with velocities elevated to $\geq 200 \mathrm{~cm} / \mathrm{s}$ should be restudied in 1 month, and studies with borderline or mildly abnormal results should be repeated in 3-6 months. Periodic transfusions to reduce the percentage of sickle hemoglobin are recommended in children with abnormal TCD results $[22 \bullet \bullet]$.

Complex Congenital Heart Disease Presents Risk for Stroke in the Peri-Operative Period, but Stroke Risk Persists for Many Years After Surgery

Congenital heart disease and other cardiac problems are still recognized as presumed risk factors for childhood stroke because of their high frequency in case series [2]. A case-control study of children in northern California demonstrated an increased risk for children with congenital heart disease requiring surgical repair $(\mathrm{OR}=11 ; 95 \%$ CI, 3.1-39.4) (Fox and Fullerton, unpublished data). Even with modern surgical techniques, the peri-operative period may be of particularly high risk, as demonstrated in a recent series of 122 children undergoing open heart surgery, which identified stroke in $10 \%$ [22••]. However, children with complex congenital heart disease remain at risk for years after their last surgery, which reflects increased stroke risk from the heart disease itself. When feasible, complex congenital heart lesions should be repaired to improve cardiac function and reduce the risk of subsequent stroke [22••].

Prothrombotic Disorders Increase the Risk of Stroke in the Setting of Additional Risk Factors

Finally, controversies remain over the strength of association of hypercoaguable disorders as a risk for childhood stroke. Homozygosity for MTHFR C677T polymorphism did not show an increased risk for arterial ischemic stroke in two recent studies of stroke in children in the United States [24] and Croatia [25], but a study in Poland [26] found a threefold increased risk of stroke with the presence of the MTHFR C677T allele. Factor V G1691A was associated with a sevenfold increased risk among children with stroke in the Croatian study [25]. Although various coagulation abnormalities have been reported in children after stroke, most pediatric stroke experts believe the likelihood of stroke from most prothrombotic states seems 
to be relatively low. However, the risk may tend to increase in the setting of additional risk factors. Children with a stroke who are found to have a prothrombotic polymorphism or other prothrombotic state should have additional workup including serial vascular imaging to determine etiology. Although there are no class I recommendations regarding hypercoaguable states in childhood stroke, it is reasonable to evaluate for elevated serum homocysteine levels and the more common prothrombotic states, and start folate, vitamin $\mathrm{B}_{6}$, or vitamin $\mathrm{B}_{12}$ if homocysteine is found to be elevated. It is also reasonable to discontinue oral contraceptives in adolescents after an ischemic or thrombotic stroke [22].

\section{Management of Pediatric Stroke: Need for Further Study}

Acute management of children with stroke is primarily supportive, including control of fever, blood pressure normalization to accepted age ranges, normalization of glucose, and maintenance of normal oxygenation. For secondary stroke prevention, the majority of children are treated with either anticoagulation or antiplatelet therapies, and current practice varies widely. Overall, the great variety of treatment strategies emphasizes the need for better evidence and clinical trials to guide management of childhood stroke.

\section{Antiplatelet Agents or Anticoagulation are Used for Secondary Stroke Prevention}

Of the children enrolled in the IPSS who had a nonneonatal arterial ischemic stroke, $27 \%$ of children were treated with anticoagulation alone, $28 \%$ were treated with antiplatelet therapy alone, and $16 \%$ were treated with both anticoagulation and antiplatelet therapies [27]. Predictors of anticoagulation were dissection and cardiac disease. This parallels recent American Heart Association guidelines [22*•] and American College of Chest Physicians guidelines [28••] for consideration of anticoagulation in children in the setting of confirmed cardio-embolism or arterial dissection. Because the risk for recurrence is relatively high in children who have arteriopathies, there has been some interest in exploring the safety of anticoagulation in this setting. A nonrandomized, unblinded study from the Colorado and German (COAG) collaboration examined both therapeutic and prophylactic dosing of anticoagulation therapy for stroke in children with non-moyamoya arteriopathy, with only two clinically relevant bleeding episodes in 1329 patient-months [11]. The relatively low risk of bleeding suggests that anticoagulation could be safely studied in future trials.
Clinical Trials are Required Before Tissue Plasminogen Activator is Used for Acute Stroke Treatment in Younger Ages

Although tissue plasminogen activator (tPA) has changed the face of adult stroke treatment, there have been no randomized controlled trials of therapeutics in childhood arterial ischemic stroke. A special writing group of the American Heart Association Stroke Council and the Council on Cardiovascular Disease in the Young has recently published an extensive review of the literature on childhood stroke with consensus guidelines for the management of stroke in infants and children [22••]. In that review, there was no consensus about the use of tPA in older adolescents who otherwise met standard adult tPA eligibility criteria. In younger children, tPA was not recommended outside of a clinical trial. In the IPSS observational cohort of children with acute arterial ischemic stroke, $2 \%$ of the children received either intravenous or intra-arterial tPA, at a median time of $3.3 \mathrm{~h}$ or $4.5 \mathrm{~h}$, respectively. Of these 15 patients, new or increased intracerebral hemorrhage was found in four, and the recurrent stroke rate at 1 year was 14\% [29]. In addition, a recent literature review described a series of 17 children who had received intravenous, intra-arterial, or mechanical thrombolysis for large vessel strokes; 16 children survived and 12 had a good outcome [30].

Delay in Pediatric Stroke Recognition is a Barrier to Efficient Care and Future Stroke Trials

The appropriate dosage of tPA in various age groups remains an unknown, and extrapolation from adult data is complicated by age-related differences in coagulation systems. Thus far, there are no systematic data to guide the age-appropriate use of tPA in children, and clinical trials are needed to explore safety and efficacy in this population. The Thrombolysis in Pediatric Stroke (TIPS) trial is in planning stages; it aims to test the hypothesis that tPA can be given safely in acute childhood arterial ischemic stroke and establish dosing guidelines [31]. However, conducting a clinical trial in a vulnerable population can present significant challenges. In addition, because few pediatric strokes are diagnosed quickly enough to consider a thrombolytic agent, trials of acute intervention may have slow accrual. Although public health campaigns have successfully raised awareness about stroke in adults, awareness of pediatric stroke remains limited. Children often present late to medical care, and even after medical evaluation have significant delays in diagnosis. Although the majority of children with stroke present with focal findings such as hemiparesis [32], nonspecific presenting signs such as altered mental status, seizure, and headache 
are also frequently reported. One recent study of childhood arterial ischemic stroke (excluding neonatal stroke) in Australia found a median time of greater than $24 \mathrm{~h}$ after clinical onset before radiologic confirmation of diagnosis [32]. In this study, only $6.8 \%$ of the children were diagnosed with a stroke within $3 \mathrm{~h}$. In the United Kingdom [33], Canada [34], and the United States [35], similar time delays have been reported. Nonspecific presentation and the perception that childhood stroke is rare both contribute to initial misdiagnoses that result in delays in management. Raising awareness of pediatric stroke is crucial to improving overall care of these children.

\section{Outcomes: The High Cost of Pediatric Stroke}

Pediatric stroke is an important cause of childhood morbidity and lifelong disability, including motor and sensory impairments, cognitive deficits, and epilepsy. In the IPSS cohort, after arterial ischemic stroke $74 \%$ of patients had neurologic deficits at hospital discharge and $3 \%$ of the children had died [27]. Long-term outcomes for children in the IPSS are not yet available. Although physical, occupational, and speech therapies are mainstays to improve function in children with neurologic deficits after a stroke, pediatric-specific research regarding different methods of rehabilitation is lacking.

\section{Children with Arteriopathies Maybe at Elevated Risk for Recurrent Stroke}

Overall, clinical stroke recurrences are seen in an estimated $6-15 \%$ of children after a first arterial ischemic stroke, but the risk of recurrence depends on stroke etiology. Braun et al. [36•] described a series of 79 children found to have an intracranial unilateral arteriopathy after a stroke. In 74 of the children, the arteriopathy stabilized or improved and was categorized as TCA, although $77 \%$ of these had residual vascular abnormalities. Recurrent stroke or transient ischemic attack occurred in $18 \%$ of the children categorized as TCA after a median follow-up of 1.4 years. Progressive arteriopathies were associated with recurrence $(\mathrm{OR}=18.7$; 95\% CI, 1.9-182). In the COAG cohort, children with non-moyamoya arteriopathies were given various treatments of prophylactic or therapeutic anticoagulation, and cumulative probability of recurrent stroke was $14 \%$ at 1 year [11]. These recent findings build on prior studies that found abnormal vascular imaging predicts recurrence [6]. Systematic studies of secondary stroke prevention in children with arteriopathies are not yet available, and clinical trials are needed to determine the best treatment strategies.
Epilepsy Maybe Frequent After Childhood Stroke

In comparison with the low risk of epilepsy after stroke in adults, estimated at 2-4\% [37], the risk of epilepsy after childhood stroke maybe an order of magnitude higher, with some estimates as high as $67 \%$ after a neonatal stroke [38]. Follow-up studies of stroke later in childhood give estimates of epilepsy ranging from 25 to $30 \%$ [39]. Although the frequency of epilepsy after childhood stroke reported in these case series is worrisome, the estimates of post-stroke epilepsy prevalence are wide ranging and there are few data regarding predictors of epilepsy to alert physicians to high-risk groups. More research is required to determine the incidence and predictors of epilepsy in these children.

Epilepsy after pediatric stroke is particularly worrisome because of the additional morbidities reported in children who have seizures after brain injury. After a stroke, children are already at risk for behavioral and psychiatric problems [40]. In turn, attention deficit hyperactivity disorder (ADHD) can further impair school performance, particularly in children whose cognitive functioning may already be compromised. Recent studies have suggested that children with epilepsy after a childhood stroke maybe at particular risk for behavioral and psychiatric co-morbidities such as ADHD and depression, and it may herald worse neurocognitive outcomes [41, 42]. Some researchers theorize that seizures after a stroke in the developing brain may change the brain's capacity for plasticity. However, it is not known whether the risk for poor behavioral and cognitive outcomes that we have begun to recognize in these children maybe due to presence of seizures, the medications to treat the seizures, or whether the seizures are simply a marker of inherent capacity in children with more severe brain injury.

\section{Quantitative Imaging Techniques may Provide Better} Prognostication of Stroke Outcomes in the Future

Infarct size and altered mental status at presentation predict long-term outcome of pediatric stroke [27, 43, 44]. However, these broad measures still leave physicians and families with little to guide their expectations for the future. Children with neonatal stroke may not manifest hemiparesis until months after birth, and a few studies have focused on finding earlier predictors of outcome. One study of 13 children after a neonatal stroke examined hand movements at 3 months of age. The study found asymmetric hand movements could be determined with good inter-observer agreement, and it predicted later hemiplegia in 13 children [45]. Researchers are also studying imaging techniques as a way to provide earlier prognostication. Novel imaging techniques using measurements of diffusion MRI found pre-Wallerian degeneration of corticospinal tracts to be 
predictive of poor motor outcome after neonatal [46] and pediatric [47] stroke. Although these diffusion-weighted imaging quantification techniques are not yet routinely used, in the future similar imaging techniques may provide better prognostication. A more accurate assessment of outcome at early time points after a childhood stroke would be helpful both for family expectations and for selection of patients in trials of therapeutic interventions.

\section{Childhood Stroke is Associated with High Costs of Medical Care}

In terms of the economic burden of stroke care, adult stroke has been analyzed extensively for costeffectiveness of interventions. A 2009 review of 120 cost studies of adult ischemic and hemorrhagic stroke from 15 countries found the mean cost of adult stroke was $\$ 19,018$, but varied as much as 20 -fold in the United States estimates [48]. In contrast, fewer studies have examined the cost of pediatric stroke. Two recent studies have estimated acute and long-term costs of childhood stroke in 2003 dollars. The mean cost of acute hospital care for pediatric stroke was high, estimated at $\$ 20,927$ per discharge in 2003 using a national database of ICD-9 codes (the Kids' Inpatient Database) for both ischemic and hemorrhagic stroke [49]. Hemorrhagic stroke, geographic data, urban teaching hospitals, and discharge disposition were predictors of higher cost. In a Northern California health maintenance organization, the average coast of a childhood acute stroke admission was $\$ 90,693$ for hemorrhagic stroke and $\$ 36,073$ for ischemic stroke. In the same cohort, the average cost of birth admission for a neonatal stroke was $\$ 39,613$ [50]. When costs were adjusted for a stroke-free birth admission, cost of the stroke was estimated to account for $88 \%$ of the admission cost. In addition, direct medical costs for inpatient and outpatient health services over 5 years were estimated to be $\$ 51,719$ for neonatal stroke and $\$ 135,161$ overall in the Northern California cohort. These studies suggest that the financial cost of pediatric stroke is high, and that the economic burden of medical care for these children continues for many years after the acute hospitalization.

\section{Conclusions}

Childhood arterial ischemic stroke differs from adult stroke in risk factors, etiologies, and outcomes. Recent studies have focused attention on the importance of nonatherosclerotic arteriopathies and recent infection. Although the causes of pediatric stroke are very different from adult stroke, many of the current therapeutic strategies and secondary stroke prevention with antiplatelets or antico- agulation are adapted from adult stroke management. Little is known about the safety and efficacy of acute thrombolytic therapies in various age groups. Finally, long-term outcomes of pediatric stroke are difficult to predict, but overall place a large burden on stroke victims, families, and society. Further study is greatly needed for a better understanding of the pathogenesis, management, and outcomes in childhood arterial ischemic stroke.

Open Access This article is distributed under the terms of the Creative Commons Attribution Noncommercial License which permits any noncommercial use, distribution, and reproduction in any medium, provided the original author(s) and source are credited.

\section{References}

Papers of particular interest, published recently, have been highlighted as:

- Of importance

•- Of major importance

1. Agrawal N, Johnston $\mathrm{SC}, \mathrm{Wu} \mathrm{YW}$, et al. Imaging data reveal a higher pediatric stroke incidence than prior US estimates. Stroke 2009;40:3415-3421.

2. Lo W, Stephens J, Fernandez S. Pediatric stroke in the United States and the impact of risk factors. J Child Neurol 2009;24:194-203.

3. Golomb MR, Fullerton HJ, Nowak-Gottl U, Deveber G. Male predominance in childhood ischemic stroke: findings from the international pediatric stroke study. Stroke 2009;40:52-57.

4. Normann S, de Veber G, Fobker M, et al. Role of endogenous testosterone concentration in pediatric stroke. Ann Neurol 2009;66:754-758.

5. Mackay M, Wiznitzer M, Benedict S, et al. Risk factors for childhood arterial ischemic stroke: results from the International Pediatric Stroke Study [abstract]. Presented at International Stroke Conference 2010. San Antonio, TX; February 24-26, 2010.

6. Fullerton HJ, Wu YW, Sidney S, Johnston SC. Risk of recurrent childhood arterial ischemic stroke in a population-based cohort: the importance of cerebrovascular imaging. Pediatrics 2007;119:495-501.

7. - Amlie-Lefond C, Bernard TJ, Sebire G, et al. Predictors of cerebral arteriopathy in children with arterial ischemic stroke: results of the International Pediatric Stroke Study. Circulation 2009;119:1417-1423 Results from the International Pediatric Stroke Study of 525 children with vascular imaging after an arterial ischemic stroke demonstrate the importance of arteriopathies. The term "focal cerebral arteriopathy" is suggested to describe the common finding of focal cerebral arterial stenosis with no apparent cause. Prior upper respiratory infection was associated with focal cerebral arteriopathy, supporting the role of infection in childhood stroke.

8. Rea D, Brandsema JF, Armstrong D, et al. Cerebral arteriopathy in children with neurofibromatosis type 1. Pediatrics 2009 (in press).

9. Chabrier S, Rodesch G, Lasjaunias P, et al. Transient cerebral arteriopathy: a disorder recognized by serial angiograms in children with stroke. J Child Neurol 1998;13:27-32.

10. Ganesan V, Chong WK, Cox TC, et al. Posterior circulation stroke in childhood: risk factors and recurrence. Neurology 2002;59:15521556. 
11. Bernard TJ, Goldenberg NA, Tripputi M, et al. Anticoagulation in childhood-onset arterial ischemic stroke with non-moyamoya arteriopathy: findings from the Colorado and German (COAG) collaboration. Stroke 2009;40:2869-2871.

12. Nagel MA, Cohrs RJ, Mahalingam R, et al. The varicella zoster virus vasculopathies: clinical, CSF, imaging, and virologic features. Neurology 2008;70:853-860.

13. Miravet E, Danchaivijitr N, Basu H, et al. Clinical and radiological features of childhood cerebral infarction following varicella zoster virus infection. Dev Med Child Neurol 2007;49:417-422.

14. Donahue JG, Kieke BA, Yih WK, et al. Varicella vaccination and ischemic stroke in children: is there an association? Pediatrics 2009;123:e228-e234.

15. Ohene-Frempong K, Weiner SJ, Sleeper LA, et al. Cerebrovascular accidents in sickle cell disease: rates and risk factors. Blood 1998;91:288-294.

16. Adams R, McKie V, Nichols F, et al. The use of transcranial ultrasonography to predict stroke in sickle cell disease. N Engl J Med 1992;326:605-610.

17. Adams RJ, McKie VC, Hsu L, et al. Prevention of a first stroke by transfusions in children with sickle cell anemia and abnormal results on transcranial Doppler ultrasonography. N Engl J Med 1998;339:5-11.

18. Mirre E, Brousse V, Berteloot L, et al. Feasibility and efficacy of chronic transfusion for stroke prevention in children with sickle cell disease. Eur J Haematol 2005;84:259-265.

19. Colombatti R, Meneghetti G, Ermani M, et al. Primary stroke prevention for sickle cell disease in north-east Italy: the role of ethnic issues in establishing a Transcranial Doppler screening program. Ital J Pediatr 2009;35:15.

20. Armstrong-Wells J, Grimes B, Sidney S, et al. Utilization of TCD screening for primary stroke prevention in children with sickle cell disease. Neurology 2009;72:1316-1321.

21. Dowling MM, Lee N, Quinn CT, et al. Prevalence of intracardiac shunting in children with sickle cell disease and stroke. J Pediatr 2009 (in press).

22. • Roach ES, Golomb MR, Adams R, et al. Management of stroke in infants and children: a scientific statement from a Special Writing Group of the American Heart Association Stroke Council and the Council on Cardiovascular Disease in the Young. Stroke 2008;39:2644-2691 The American Heart Association Stroke counsel provides a statement of evidencebased recommendations for management of pediatric stroke in various subpopulations of children, including perinatal stroke, childhood arterial ischemic stroke, sickle cell disease, moyamoya disease, cervicocephalic arterial dissection, and cardiogenic embolism. Protocols for dosing of heparin and warfarin in children are suggested.

23. Chen J, Zimmerman RA, Jarvik GP, et al. Perioperative stroke in infants undergoing open heart operations for congenital heart disease. Ann Thorac Surg 2009;88:823-829.

24. Morita DC, Donaldson A, Butterfield RJ, et al. Methylenetetrahydrofolate reductase gene polymorphism and childhood stroke. Pediatr Neurol 2009;41:247-249.

25. Herak DC, Antolic MR, Krleza JL, et al. Inherited prothrombotic risk factors in children with stroke, transient ischemic attack, or migraine. Pediatrics 2009;123:e653-e660.

26. Zak I, Sarecka-Hujar B, Kopyta I, et al. The T allele of the $677 \mathrm{C}>\mathrm{T}$ polymorphism of methylenetetrahydrofolate reductase gene is associated with an increased risk of ischemic stroke in Polish children. J Child Neurol 2009;24:1262-1267.

27. Goldenberg NA, Bernard TJ, Fullerton HJ, et al. Antithrombotic treatments, outcomes, and prognostic factors in acute childhoodonset arterial ischaemic stroke: a multicentre, observational, cohort study. Lancet Neurol 2009;8:1120-1127.
28. •- Monagle P, Chalmers E, Chan A, et al. Antithrombotic therapy in neonates and children: American College of Chest Physicians Evidence-Based Clinical Practice Guidelines (8th Edition). Chest 2008;133:887S-968S The American College of Chest Physicians provides clinical practice guidelines for antithrombotic therapy in neonates and children, including guidelines for treatment of acute arterial ischemic stroke with heparin or aspirin.

29. Amlie-Lefond C, deVeber G, Chan AK, et al. Use of alteplase in childhood arterial ischaemic stroke: a multicentre, observational, cohort study. Lancet Neurol 2009;8:530-536.

30. Arnold M, Steinlin M, Baumann A, et al. Thrombolysis in childhood stroke: report of 2 cases and review of the literature. Stroke 2009;40:801-807.

31. Amlie-Lefond C, Chan AK, Kirton A, et al. Thrombolysis in acute childhood stroke: design and challenges of the thrombolysis in pediatric stroke clinical trial. Neuroepidemiology 2009;32:279-286

32. Srinivasan J, Miller SP, Phan TG, Mackay MT. Delayed recognition of initial stroke in children: need for increased awareness. Pediatrics 2009; 124:e227-e234.

33. McGlennan C, Ganesan V. Delays in investigation and management of acute arterial ischaemic stroke in children. Dev Med Child Neurol 2008;50:537-540.

34. Rafay MF, Pontigon AM, Chiang J, et al. Delay to diagnosis in acute pediatric arterial ischemic stroke. Stroke 2009;40:58 64.

35. Hartman AL, Lunney KM, Serena JE. Pediatric stroke: do clinical factors predict delays in presentation? J Pediatr 2009; 154:727-732.

36. Braun KP, Bulder MM, Chabrier S, et al. The course and outcome of unilateral intracranial arteriopathy in 79 children with ischaemic stroke. Brain 2009;132:544-557 The evolution of vascular imaging after childhood arterial ischemic stroke is described in a European cohort. Clinical and imaging characteristics are compared in transient versus progressive cerebral arteriopathies. The majority of children fell under the category of transient cerebral arteriopathy, and recurrent stroke was associated with progressive cerebrovascular disease.

37. Camilo O, Goldstein LB. Seizures and epilepsy after ischemic stroke. Stroke 2004;35:1769-1775.

38. Golomb MR, Garg BP, Carvalho KS, et al. Perinatal stroke and the risk of developing childhood epilepsy. J Pediatr 2007;151:409-413, 13e1-2.

39. Lee JC, Lin KL, Wang HS, et al. Seizures in childhood ischemic stroke in Taiwan. Brain Dev 2009;31:294-299.

40. Everts R, Pavlovic J, Kaufmann F, et al. Cognitive functioning, behavior, and quality of life after stroke in childhood. Child Neuropsychol 2008;14:323-338.

41. Ricci D, Mercuri E, Barnett A, et al. Cognitive outcome at early school age in term-born children with perinatally acquired middle cerebral artery territory infarction. Stroke 2008;39:403410.

42. Ballantyne AO, Spilkin AM, Hesselink J, Trauner DA. Plasticity in the developing brain: intellectual, language and academic functions in children with ischaemic perinatal stroke. Brain 2008;131:2975-2985.

43. Beslow LA, Licht DJ, Smith SE, et al. Predictors of outcome in childhood intracerebral hemorrhage: a prospective consecutive cohort study. Stroke 2009;41:313-318.

44. Jordan LC, Kleinman JT, Hillis AE. Intracerebral hemorrhage volume predicts poor neurologic outcome in children. Stroke 2009;40:1666-1671.

45. Guzzetta A, Pizzardi A, Belmonti V, et al. Hand movements at 3 months predict later hemiplegia in term infants with 
neonatal cerebral infarction. Dev Med Child Neurol 2009 (in press).

46. Kirton A, Shroff M, Visvanathan T, deVeber G. Quantified corticospinal tract diffusion restriction predicts neonatal stroke outcome. Stroke 2007;38:974-980.

47. Domi T, deVeber G, Shroff M, et al. Corticospinal tract prewallerian degeneration: a novel outcome predictor for pediatric stroke on acute MRI. Stroke 2009;40:780-787.
48. Luengo-Fernandez R, Gray AM, Rothwell PM. Costs of stroke using patient-level data: a critical review of the literature. Stroke 2009;40:e18-e23.

49. Perkins E, Stephens J, Xiang H, Lo W. The cost of pediatric stroke acute care in the United States. Stroke 2009;40:2820-2827.

50. Gardner MA, Hills NK, Sidney S, et al. The 5-year direct medical cost of neonatal and childhood stroke in a population-based cohort. Neurology 2009;74:372-378. 